\title{
Note on Coupled Linear Systems Related to Two Soliton Collision for the Quartic gKdV Equation
}

\author{
Yvan MARTEL and Frank MerLe \\ Université de Versailles Saint-Quentin-en-Yvelines \\ Mathématiques \\ 45, av. des Etats-Unis \\ 78035 Versailles cedex - France \\ martel@math.uvsq.fr \\ Université de Cergy-Pontoise \\ IHES and CNRS, Mathématiques \\ 2, av. Adolphe Chauvin \\ 95302 Cergy-Pontoise cedex - France \\ Frank.Merle@math.u-cergy.fr
}

Received: June 19, 2007

Accepted: October 29, 2007

\section{ABSTRACT}

We consider linear systems related to the description of the collision of two solitons for the gKdV equation with quartic nonlinearity. The computations presented in this note are applied in Martel and Merle [10] to prove a result concerning inelastic (but almost elastic) collision for a nonintegrable equation.

Key words: soliton, collision, gKdV equation.

2000 Mathematics Subject Classification: 35Q53, 35Q51, 35B40.

\section{Introduction}

We consider the quartic generalized Korteweg-de Vries (gKdV) equation:

$$
\partial_{t} u+\partial_{x}\left(\partial_{x}^{2} u+u^{4}\right)=0, \quad x, t \in \mathbb{R} .
$$

The objective of this note is to solve some linear coupled systems related to the collision of two solitons for (1) (see also section 2 for the case where the nonlinearity is $u^{2}$ ). Recall that equation (1), unlike the corresponding equations with nonlinearities $u^{2}$ or $u^{3}$, is not integrable. In particular, the collision of two solitary waves of (1)

This research was supported in part by the Agence Nationale de la Recherche (ANR ONDENONLIN). 
is not described by explicit 2-soliton solutions. In [10], we have introduced a new method to understand the collision of two solitary waves of (1) in the special case where one solitary wave is small with respect to the other. More precisely, denote by $Q$ the unique even solution of

$$
Q>0, \quad Q^{\prime \prime}+Q^{4}=Q, \quad Q \in H^{1}(\mathbb{R}), \quad \text { i.e. }, \quad Q(x)=\left(\frac{5}{2 \cosh ^{2}\left(\frac{3}{2} x\right)}\right)^{\frac{1}{3}}
$$

and, for any $c>0$, let $Q_{c}(x)=c^{\frac{1}{3}} Q(\sqrt{c} x)$ be a solution of $Q_{c}^{\prime \prime}+Q_{c}^{4}=c Q_{c}$. Then, for any $x_{0} \in \mathbb{R}, c>0$, the functions

$$
R_{c, x_{0}}(t, x)=Q_{c}\left(x-x_{0}-c t\right)
$$

are solitary wave solutions of (1), called solitons.

From [7] (see also [8]), there exist solutions $u(t, x)$ of $(1)$ which are asymptotic $N$-soliton solutions at $t \rightarrow-\infty$ in the following sense: let $N \geq 1, c_{1}>\cdots>c_{N}>0$, and $x_{1}, \ldots, x_{N} \in \mathbb{R}$, there exists a unique $H^{1}$ solution $U$ of (1) such that

$$
\lim _{t \rightarrow-\infty}\left\|U(t)-\sum_{j=1}^{N} Q_{c_{j}}\left(\cdot-x_{j}-c_{j} t\right)\right\|_{H^{1}(\mathbb{R})}=0 .
$$

The behavior displayed by these solutions is stable in some sense. Considering for example the case of two solitons, there exist a large class of solutions such that, as $t \sim-\infty$,

$$
u(t, x)=Q_{c_{1}}\left(x-x_{1}-c_{1} t\right)+Q_{c_{2}}\left(x-x_{2}-c_{2} t\right)+\eta(t, x),
$$

where $c_{1}>c_{2}$ and $\eta(t)$ is a dispersion term small in the energy space $H^{1}$ with respect to $Q_{c_{1}}, Q_{c_{2}}$ (see [8]). From the Physics point of view, the two solitons $Q_{c_{1}}$ and $Q_{c_{2}}$ have to collide at some time $t_{0}$. In the special case $c_{2} \ll c_{1}$ (or equivalently, $\left.\left\|Q_{c_{2}}\right\|_{H^{1}} \ll\left\|Q_{c_{1}}\right\|_{H^{1}}\right)$ and $\|\eta(t)\|_{H^{1}} \ll\left\|Q_{c_{2}}\right\|_{H^{1}}$, for $t$ close to $-\infty$, we have introduced in [10] explicit computations allowing to understand the collision at the main orders, using a new nonlinear "basis" to write and compute an approximate solution $v(t, x)$ up to any order of size.

Recall that the problem of collision of two solitons is a classical question in nonlinear wave propagation (see $[3,13,14]$ ). In the so-called integrable cases (i.e., when the nonlinearity in (1) is $u^{2}$ or $u^{3}$ ) it is well-known that there exist explicit multi-soliton solutions, describing the elastic collision of several solitons (see Hirota [4], Lax [5], Wadati and Toda [12], and the review paper Miura [11]). Note that in experiments, or numerically for more accurate nonintegrable models (see Craig et al. [2], Li and Sattinger [6], Bona et al. [1], and other references in [10]), this remarkable property is mainly preserved, i.e., the collision of two solitons is almost elastic; however, a (very small) residual part is observed after the collision. Equation (1) being not integrable, explicit $N$-soliton solutions are not available in this case. The results obtained in [10], 
using Theorem A of the present paper, are the first rigorous results concerning inelastic (but almost elastic) collision in a nonintegrable situation. We refer to the introduction and the references in [10] for a overview on these questions.

The approximate solution $v(t, x)$ in [10] has the following structure: let $k_{0} \geq 1$, $\ell_{0} \geq 0$, and

$$
\Sigma_{0}=\left\{(k, \ell), \quad 1 \leq k \leq k_{0}, 0 \leq \ell \leq \ell_{0}\right\} .
$$

We set

$$
\begin{gathered}
y_{c}=x+(1-c) t \quad \text { and } \quad R_{c}(t, x)=Q_{c}\left(y_{c}\right), \\
y=x-\alpha\left(y_{c}\right) \quad \text { and } \quad R(t, x)=Q(y),
\end{gathered}
$$

where for $\left(a_{k, \ell}\right)_{(k, \ell) \in \Sigma_{0}}$

$$
\alpha(s)=\int_{0}^{s} \beta\left(s^{\prime}\right) d s^{\prime}, \quad \beta(s)=\sum_{(k, \ell) \in \Sigma_{0}} a_{k, \ell} c^{\ell} Q_{c}^{k}(s) .
$$

The form of $v(t, x)$ is

$$
\begin{aligned}
v(t, x) & =Q(y)+Q_{c}\left(y_{c}\right)+W(t, x), \\
W(t, x) & =\sum_{(k, \ell) \in \Sigma_{0}} c^{\ell}\left(Q_{c}^{k}\left(y_{c}\right) A_{k, \ell}(y)+\left(Q_{c}^{k}\right)^{\prime}\left(y_{c}\right) B_{k, \ell}(y)\right),
\end{aligned}
$$

where $a_{k, \ell}, A_{k, \ell}, B_{k, \ell}$ are to be determined. Let $S(t)=\partial_{t} v+\partial_{x}\left(\partial_{x}^{2} v-v+v^{4}\right)$. We have obtained in [10, Proposition 2.1]:

$$
\begin{aligned}
S(t, x)= & -\sum_{(k, \ell) \in \Sigma_{0}} c_{c}^{\ell} Q_{c}^{k}\left(y_{c}\right)\left(\left(\mathcal{L} A_{k, \ell}\right)^{\prime}+a_{k, \ell}\left(3 Q-2 Q^{4}\right)^{\prime}-F_{k, \ell}\right) r(y) \\
& -\sum_{(k, \ell) \in \Sigma_{0}} c^{\ell}\left(Q_{c}^{k}\right)^{\prime}\left(y_{c}\right)\left(\left(\mathcal{L} B_{k, \ell}\right)^{\prime}+a_{k, \ell}\left(3 Q^{\prime \prime}\right)-\left(3 A_{k, \ell}^{\prime \prime}+4 Q^{3} A_{k, \ell}\right)-G_{k, \ell}\right)(y) \\
& +\mathcal{E}(t, x),
\end{aligned}
$$

where

$$
\mathcal{L} w=-\partial_{x}^{2} w+w-4 Q^{3} w,
$$

$\mathcal{E}(t, x)$ is a lower order term and the functions $F_{k, \ell}, G_{k, \ell}$ are second member terms in the sense that they depend only on $\left(a_{k^{\prime}, \ell^{\prime}}, A_{k^{\prime}, \ell^{\prime}}, B_{k^{\prime}, \ell^{\prime}}\right)$, for $\left(k^{\prime}, \ell^{\prime}\right)$ such that $k^{\prime}<k$, $\ell^{\prime} \leq \ell$, or $k^{\prime} \leq k, \ell^{\prime}<\ell$. Therefore, to obtain a suitable approximate solution (i.e., for which $S=\mathcal{E}$ ), we want to solve by induction on $(k, \ell)$ the following systems:

$$
\left(\Omega_{k, \ell}\right) \quad\left\{\begin{array}{l}
\left(\mathcal{L} A_{k, \ell}\right)^{\prime}+a_{k, \ell}\left(3 Q-2 Q^{4}\right)^{\prime}=F_{k, \ell} \\
\left(\mathcal{L} B_{k, \ell}\right)^{\prime}+a_{k, \ell}\left(3 Q^{\prime \prime}\right)-3 A_{k, \ell}^{\prime \prime}-4 Q^{3} A_{k, \ell}=G_{k, \ell} .
\end{array}\right.
$$


We have proved in [10] that for a certain structure of $F_{k, \ell}, G_{k, \ell}$, one could always solve the system $(\Omega)$, see [10, Propositions 2.2 and 2.3].

Using this approximate solution $v$, and analysis arguments (allowing to relate $v(t)$ to solutions $u(t)$ of $(1)$ ), we have proved the following result in [10, Theorem 1].

Non existence of a pure 2-soliton [10]. Let $c>0$. There exists $\epsilon_{0}>0$ such that, if $c<\epsilon_{0}$, then there exists no pure 2-soliton solution with speeds 1 and $c$. More precisely, let $u(t)$ be the unique $H^{1}$ solution of (1) such that

$$
\lim _{t \rightarrow-\infty}\left\|u(t)-Q(\cdot-t)-Q_{c}(\cdot-c t)\right\|_{H^{1}}=0 .
$$

Then, there exist $x_{1}^{+}, x_{2}^{+}, c_{1}^{+}>c_{2}^{+}>0$, and $K>0$ such that

$$
w^{+}(t, x)=u(t, x)-Q_{c_{1}^{+}}\left(x-x_{1}^{+}-c_{1}^{+} t\right)-Q_{c_{2}^{+}}\left(x-x_{2}^{+}-c_{2}^{+} t\right)
$$

satisfies

$$
\begin{gathered}
\lim _{t \rightarrow+\infty}\left\|w^{+}(t)\right\|_{H^{1}\left(x>\frac{1}{10} c t\right)}=0, \\
\frac{1}{K} c^{\frac{17}{6}} \leq c_{1}^{+}-1 \leq K c^{\frac{11}{12}}, \quad \frac{1}{K} c^{\frac{8}{3}} \leq 1-\frac{c_{2}^{+}}{c} \leq K c^{\frac{1}{3}}, \\
\frac{1}{K} c^{\frac{17}{12}} \leq\left\|\partial_{x} w^{+}(t)\right\|_{L^{2}}+\sqrt{c_{1} c}\left\|w^{+}(t)\right\|_{L^{2}} \leq K c^{\frac{11}{12}}, \quad \text { for } t \text { large. }
\end{gathered}
$$

The lower bound in (4) gives the minimal distance of $u(t)$ to a two soliton solution after the collision. It is thus a qualitative version of the nonexistence of pure 2-soliton solution. Estimate (3) means that the speed of faster soliton is increased and the speed of the smaller one is decreased due to the collision.

For the proof of this result in [10], we needed in particular the resolution of $\left(\Omega_{1,0}\right)$ and the value of $b_{2,0}=\lim _{x \rightarrow+\infty} B_{2,0}(x)$ in $\left(\Omega_{2,0}\right)$. Let $\mathcal{Y}$ be the set of functions $f \in C^{\infty}(\mathbb{R})$ such that

$$
\forall j \in \mathbb{N}, \quad \exists K_{j}, r_{j}>0, \quad \forall x \in \mathbb{R}, \quad\left|f^{(j)}(x)\right| \leq K_{j}(1+|x|)^{r_{j}} e^{-|x|} .
$$

The first system $\left(\Omega_{1,0}\right)$ has been solved in [10, Proposition 2.3 and Lemma 3.1]. We recall

$$
\left(\Omega_{1,0}\right) \quad\left\{\begin{array}{l}
\mathcal{L} A_{1,0}+a_{1,0}\left(3 Q-2 Q^{4}\right)=4 Q^{3} \\
\left(\mathcal{L} B_{1,0}\right)^{\prime}+a_{1,0}\left(3 Q^{\prime \prime}\right)-3 A_{1,0}^{\prime \prime}-4 Q^{3} A_{1,0}=4 Q^{3} .
\end{array}\right.
$$

We found a solution $\left(a_{1,0}, A_{1,0}, B_{1,0}\right)$ of $\left(\Omega_{1,0}\right)$ such that $A_{1,0}$ is even, $B_{1,0}$ is odd, and

$$
\begin{aligned}
& A_{1,0}=\frac{1}{3}\left(Q^{\prime}\left(\int_{0}^{x} Q^{2}\right)-2 Q^{3}\right)+2 \frac{\int Q}{\int Q^{2}}\left(-\frac{1}{3} Q-\frac{3}{2} x Q^{\prime}\right) \in \mathcal{Y}, \\
& B_{1,0}=\bar{B}_{1,0}+b_{1,0}\left(-\frac{Q^{\prime}}{Q}\right), \quad \bar{B}_{1,0} \in \mathcal{Y}, \\
& a_{1,0}=-2 \frac{\int Q}{\int Q^{2}}<0, \quad b_{1,0}=\lim _{x \rightarrow+\infty} B_{1,0}=-\frac{1}{2} \int Q^{3}+\frac{1}{6} \frac{\left(\int Q\right)^{2}}{\int Q^{2}}<0 .
\end{aligned}
$$


(Note that by explicit computations $\lim _{ \pm \infty}\left(-\frac{Q^{\prime}}{Q}\right)= \pm 1$.) The interpretation of the resolution of the first system is the following: $a_{1,0}$ is related to the shift on the first soliton due to the collision and $b_{1,0}$ is related to the shift on the second soliton. In particular, in contrast with the cases of the nonlinearities $u^{2}$ and $u^{3}$, the shifts on $Q$ and $Q_{c}$ are both negative, see [10, Remark 2]. Note that the explicit computation of $\bar{B}_{1,0}$ is possible but it is not required in [10].

For the system $\left(\Omega_{2,0}\right)$, which writes as follows (see [10, Proposition 2.1]):

$$
\left(\Omega_{2,0}\right)\left\{\begin{aligned}
\left(\mathcal{L} A_{2,0}\right)^{\prime}+a_{2,0}\left(3 Q-2 Q^{4}\right)^{\prime}= & \left(6 Q^{2}\left(1+A_{1,0}\right)^{2}\right)^{\prime} \\
& -a_{1,0}\left(4 Q^{3}+3 A_{1,0}^{\prime \prime}+4 Q^{3} A_{1,0}\right)^{\prime}+3 a_{1,0}^{2} Q^{(3)}, \\
\left(\mathcal{L} B_{2,0}\right)^{\prime}+3 a_{2,0} Q^{\prime \prime}-3 A_{2,0}^{\prime \prime}- & 4 Q^{3} A_{2,0} \\
= & 6 Q^{2}\left(1+A_{1,0}\right)^{2}+\left(6 Q^{2} B_{1,0}\left(1+A_{1,0}\right)\right)^{\prime} \\
& -\frac{1}{2} a_{1,0}\left(9 A_{1,0}^{\prime}+3 B_{1,0}^{\prime \prime}+4 Q^{3} B_{1,0}\right)^{\prime}+\frac{3}{2} a_{1,0}^{2} Q^{\prime \prime},
\end{aligned}\right.
$$

we claim the following, which is the main result of this note.

Theorem A. Let $\left(a_{1,0}, A_{1,0}, B_{1,0}\right)$ satisfy $\left(\Omega_{1,0}\right)$ and $(5)$. Then, there exists a solution $\left(a_{2,0}, A_{2,0}, B_{2,0}\right)$ of $\left(\Omega_{2,0}\right)$ such that $A_{2,0}$ is even, $B_{2,0}$ is odd, and

$$
\begin{gathered}
A_{2,0} \in \mathcal{Y}, \quad B_{2,0}=\bar{B}_{2,0}+b_{2,0}\left(-\frac{Q^{\prime}}{Q}\right), \quad \bar{B}_{2,0} \in \mathcal{Y}, \\
b_{2,0}=\lim _{+\infty} B_{2,0}=-\frac{1}{18} \int Q^{2}-\frac{3}{4} \frac{\left(\int Q\right)\left(\int Q^{3}\right)}{\int Q^{2}}-\frac{1}{18} \frac{\left(\int Q\right)^{3}}{\left(\int Q^{2}\right)^{2}}<0 .
\end{gathered}
$$

The fact that $b_{2,0} \neq 0$ is fundamental in the proof of [10, Theorem 1]. Note that the explicit computation of $b_{2,0}$ in this note does not require the complete resolution of the system $\left(\Omega_{2,0}\right)$. A main difficulty in solving systems $\left(\Omega_{k, \ell}\right)$ is to find explicit antecedents by the operator $\mathcal{L}$. In the proof of Theorem $\mathrm{A}$, we use the resolution of $\left(\Omega_{1,0}\right), b_{2,0}=\lim _{+\infty} B_{2,0}=\lim _{+\infty} \mathcal{L} B_{2,0}$ and the self-adjointness of $\mathcal{L}$ to avoid computing $a_{2,0}, A_{2,0}$, and $B_{2,0}$. The structure of operator $\mathcal{L}$ is described in Lemma 1.1 in section 1.

Formally, the fact that $b_{2,0} \neq 0$ means that for $t=T_{c}=c^{-\frac{1}{2}-\frac{1}{100}}$, (i.e., long after the collision time $t=0$ ), we have

$$
\begin{aligned}
v\left(T_{c}, x\right)-Q(y) & \sim Q_{c}\left(y_{c}\right)-b_{1,0} Q_{c}^{\prime}\left(y_{c}\right)-b_{2,0}\left(Q_{c}^{2}\right)^{\prime}\left(y_{c}\right) \\
& \sim Q_{c}\left(y_{c}-b_{1,0}\right)-b_{2,0}\left(Q_{c}^{2}\right)^{\prime}\left(y_{c}\right) .
\end{aligned}
$$

Thus, $v\left(T_{c}\right)$ does not match a two soliton solution because of the nonzero term $b_{2,0}\left(Q_{c}^{2}\right)^{\prime}\left(y_{c}\right)$ (for details, see proof of [10, Proposition 3.1]). Let $u(t, x)$ be the asymptotic 2 -soliton solution at $-\infty$, with speeds $1, c$. Then, it follows from the analysis in [10] that the size of $\left(b_{2,0} Q_{c}^{2}\right)^{\prime}$ measures the distance of $u(t, x)$ from a pure two soliton solution after the collision (some analysis arguments are required in addition to 
deal with the asymptotics in large time, see [9]). Recall that this is the first rigorous description of inelastic collision of two solitons.

The plan of this note is as follows. In section 1, we prove Theorem A. In section 2, we present some similar computations for the case $u^{2}$ in equation (1), used in [10]. The appendix contains the proof of some technical results.

\section{Proof of Theorem A}

We first recall the following elementary properties of the operator $\mathcal{L}$ (see Lemma 2.2 in [10]).

Lemma 1.1 (Properties of $\mathcal{L},[10])$. The operator $\mathcal{L}$ defined in $L^{2}(\mathbb{R})$ by

$$
\mathcal{L} f=-f^{\prime \prime}+f-4 Q^{3} f
$$

is self-adjoint and satisfies the following properties:

(i) First eigenfunction: $\mathcal{L} Q^{\frac{5}{2}}=-\frac{21}{4} Q^{\frac{5}{2}}$.

(ii) Second eigenfunction: $\mathcal{L} Q^{\prime}=0$; the kernel of $\mathcal{L}$ is $\left\{\lambda Q^{\prime}, \lambda \in \mathbb{R}\right\}$.

(iii) For any function $h \in L^{2}(\mathbb{R})$ orthogonal to $Q^{\prime}$ for the $L^{2}$ scalar product, there exists a unique function $f \in H^{2}(\mathbb{R})$ orthogonal to $Q^{\prime}$ such that $\mathcal{L} f=h$; moreover, if $h$ is even (respectively, odd), then $f$ is even (respectively, odd).

(iv) Suppose that $f \in H^{2}(\mathbb{R})$ is such that $\mathcal{L} f \in \mathcal{Y}$, then $f \in \mathcal{Y}$.

Proof of Theorem A. The existence part, with the required structure on $A_{2,0}, B_{2,0}$, was proved in [10, Proposition 2.3]. In this proof, for the sake of simplicity, we denote

$$
a_{1,0}=a_{1}, \quad A_{1,0}=A_{1}, \quad B_{1,0}=B_{1}, \quad a_{2,0}=a_{2}, \quad A_{2,0}=A_{2}, \quad B_{2,0}=B_{2} .
$$

From [10, Proposition 2.1], system $\left(\Omega_{2}\right)$ writes

$$
\left(\Omega_{2}\right)\left\{\begin{aligned}
\left(\mathcal{L} A_{2}\right)^{\prime}+a_{2}\left(3 Q-2 Q^{4}\right)^{\prime}= & \left(6 Q^{2}\left(1+A_{1}\right)^{2}\right)^{\prime} \\
& -a_{1}\left(4 Q^{3}+3 A_{1}^{\prime \prime}+4 Q^{3} A_{1}\right)^{\prime}+3 a_{1}^{2} Q^{(3)}, \\
\left(\mathcal{L} B_{2}\right)^{\prime}+3 a_{2} Q^{\prime \prime}-3 A_{2}^{\prime \prime}- & 4 Q^{3} A_{2} \\
= & 6 Q^{2}\left(1+A_{1}\right)^{2}+\left(6 Q^{2} B_{1}\left(1+A_{1}\right)\right)^{\prime} \\
& -\frac{1}{2} a_{1}\left(9 A_{1}^{\prime}+3 B_{1}^{\prime \prime}+4 Q^{3} B_{1}\right)^{\prime}+\frac{3}{2} a_{1}^{2} Q^{\prime \prime},
\end{aligned}\right.
$$

where $\left(a_{1}, A_{1}, B_{1}\right)$ satisfy $\left(\Omega_{1}\right)$ and (5). Recall also the following functions used in the resolution of the system $\left(\Omega_{1}\right)$ in [10, Lemma 3.1]:

$$
\begin{gathered}
V_{0}=-\frac{1}{3} Q-\frac{3}{2} x Q^{\prime}, \quad \mathcal{L} V_{0}=3 Q-2 Q^{4}, \\
V_{1}=\frac{1}{3}\left(Q^{\prime}\left(\int_{0}^{x} Q^{2}\right)-2 Q^{3}\right), \quad \mathcal{L} V_{1}=4 Q^{3}, \\
Z_{0}=3 Q^{\prime \prime}+3 V_{0}^{\prime \prime}+4 Q^{3} V_{0}, \quad Z_{1}=3 V_{1}^{\prime \prime}+4 Q^{3} V_{1}+4 Q^{3} .
\end{gathered}
$$


We now claim

$$
\begin{aligned}
2 b_{2}=-a_{1}\left(6 \int\right. & Q^{2}\left(1+A_{1}\right)^{2} V_{0}-a_{1} \int\left(\mathcal{L} B_{1}\right)^{\prime} V_{0}-\frac{9}{2} a_{1} \int A_{1} Q^{\prime \prime}+\frac{3}{2} a_{1}^{2} \int Q^{\prime \prime} Q \\
+ & \left.\frac{1}{2} \int A_{1}\left(\mathcal{L} B_{1}\right)^{\prime}\right)+6 \int Q^{2}\left(1+A_{1}\right)^{2}\left(1+V_{1}\right)-a_{1} \int\left(\mathcal{L} B_{1}\right)^{\prime} V_{1},
\end{aligned}
$$

Proof of (6). Denote by $V_{2}$ the (even) solution of

$$
\mathcal{L} V_{2}=6 Q^{2}\left(1+A_{1}\right)^{2}-a_{1}\left(4 Q^{3}+3 A_{1}^{\prime \prime}+4 Q^{3} A_{1}\right)+3 a_{1}^{2} Q^{\prime \prime} .
$$

Then, $A_{2}=V_{2}-a_{2} V_{0}$ is solution of the first line of the system $\left(\Omega_{2}\right)$. Moreover, since $\left(\mathcal{L} B_{1}\right)^{\prime}=4 Q^{3}-3 a_{1} Q^{\prime \prime}+3 A_{1}^{\prime \prime}+4 Q^{3} A_{1}$,

$$
\mathcal{L} V_{2}=6 Q^{2}\left(1+A_{1}\right)^{2}-a_{1}\left(\mathcal{L} B_{1}\right)^{\prime} .
$$

Recall from [10, Proposition 2.3] that the functions $A_{2}, B_{2}$ have the following structure: $A_{2} \in \mathcal{Y}$ is even and $B_{2}=\bar{B}_{2}+b_{2} \varphi$ is odd, where $\bar{B}_{2} \in \mathcal{Y}$ and $\lim _{ \pm \infty} \varphi= \pm 1$, $\varphi^{\prime} \in \mathcal{Y}\left(\varphi=-\frac{Q^{\prime}}{Q}\right.$, see [10, Claim 2.1]). Thus,

$$
2 b_{2}=\lim _{+\infty} B_{2,0}-\lim _{-\infty} B_{2,0}=\lim _{+\infty} \mathcal{L} B_{2,0}-\lim _{-\infty} \mathcal{L} B_{2,0}=\int\left(\mathcal{L} B_{2}\right)^{\prime} .
$$

Therefore, from the second line of $\left(\Omega_{2}\right)$, and then by $(7)$, we obtain

$$
\begin{aligned}
2 b_{2} & =4 \int Q^{3} A_{2}+6 \int Q^{2}\left(1+A_{1}\right)^{2}=4 \int Q^{3} V_{2}-4 a_{2} \int Q^{3} V_{0}+6 \int Q^{2}\left(1+A_{1}\right)^{2} \\
& =\int\left(\mathcal{L} V_{2}\right) V_{1}-4 a_{2} \int Q^{3} V_{0}+6 \int Q^{2}\left(1+A_{1}\right)^{2} \\
& =6 \int Q^{2}\left(1+A_{1}\right)^{2}\left(1+V_{1}\right)-a_{1} \int\left(\mathcal{L} B_{1}\right)^{\prime} V_{1}-4 a_{2} \int Q^{3} V_{0} .
\end{aligned}
$$

Since $V_{0}=-\frac{1}{3} Q-\frac{3}{2} x Q^{\prime}$ and $\int Q^{4}=\int Q$, we have

$$
-4 \int Q^{3} V_{0}=\frac{4}{3} \int Q^{4}+6 \int x Q^{\prime} Q^{3}=-\frac{1}{6} \int Q=\frac{1}{12} a_{1} \int Q^{2} .
$$

Thus,

$$
2 b_{2}=\left(\frac{1}{12} \int Q^{2}\right) a_{1} a_{2}+6 \int Q^{2}\left(1+A_{1}\right)^{2}\left(1+V_{1}\right)-a_{1} \int\left(\mathcal{L} B_{1}\right)^{\prime} V_{1} .
$$

Now, we determine $a_{2}$ from the equation of $B_{2}$, where we have replaced $A_{2}=V_{2}-a_{2} V_{0}$,

$$
\begin{aligned}
\left(\mathcal{L} B_{2}\right)^{\prime}+a_{2} Z_{0}=3 V_{2}^{\prime \prime}+4 Q^{3} V_{2}+6 Q^{2} & \left(1+A_{1}\right)^{2}-\frac{9}{2} a_{1} A_{1}^{\prime \prime}+\frac{3}{2} a_{1}^{2} Q^{\prime \prime} \\
& -\frac{1}{2} a_{1}\left(3 B_{1}^{\prime \prime}+4 Q^{3} B_{1}\right)^{\prime}+6\left(Q^{2}\left(1+A_{1}\right) B_{1}\right)^{\prime} .
\end{aligned}
$$


Multiplying by $Q$, integrating, and using $\mathcal{L} Q^{\prime}=0$ and

$$
\int Z_{0} Q=-\frac{1}{12} \int Q^{2}
$$

(see proof of (9) at the end of appendix A), we obtain

$$
\begin{aligned}
&-\frac{1}{12}\left(\int Q^{2}\right) a_{2} \\
&=\int\left(3 V_{2}^{\prime \prime}+4 Q^{3} V_{2}\right) Q+6 \int Q^{3}\left(1+A_{1}\right)^{2}-\frac{9}{2} a_{1} \int A_{1}^{\prime \prime} Q+\frac{3}{2} a_{1}^{2} \int Q^{\prime \prime} Q \\
&+\int\left[\frac{1}{2} a_{1}\left(3 B_{1}^{\prime \prime}+4 Q^{3} B_{1}\right)-6 Q^{2} B_{1}-6 Q^{2} A_{1} B_{1}\right] Q^{\prime} \\
&= \int V_{2}\left(3 Q^{\prime \prime}+4 Q^{4}\right)+6 \int Q^{3}\left(1+A_{1}\right)^{2}-\frac{9}{2} a_{1} \int A_{1}^{\prime \prime} Q+\frac{3}{2} a_{1}^{2} \int Q^{\prime \prime} Q \\
&+\int\left[\frac{1}{2} a_{1}\left(3 Q^{\prime \prime \prime}+4 Q^{3} Q^{\prime}\right)-6 Q^{2} Q^{\prime}-6 Q^{2} A_{1} Q^{\prime}\right] B_{1} .
\end{aligned}
$$

On the one hand,

$$
3 Q^{\prime \prime}+4 Q^{4}=3 Q+Q^{4}=\mathcal{L}\left(V_{0}-Q\right)
$$

since $\mathcal{L} Q=-3 Q^{4}$. On the other hand, since $\mathcal{L} A_{1}=4 Q^{3}-a_{1}\left(3 Q^{\prime \prime}+Q^{4}\right)$,

$$
\begin{aligned}
\frac{1}{2} a_{1}\left(3 Q^{\prime \prime \prime}+4 Q^{3} Q^{\prime}\right)-6 Q^{2} Q^{\prime}-6 Q^{2} A_{1} Q^{\prime} & =-\frac{1}{2}\left(\mathcal{L} A_{1}\right)^{\prime}-\frac{1}{2} A_{1}\left(4 Q^{3}\right)^{\prime} \\
& =-\frac{1}{2}\left(\left(\mathcal{L} A_{1}\right)^{\prime}+4\left(Q^{3}\right)^{\prime} A_{1}\right)=-\frac{1}{2} \mathcal{L}\left(A_{1}^{\prime}\right) .
\end{aligned}
$$

Thus, by (7), since $\mathcal{L} Q^{\prime}=0$,

$$
\begin{aligned}
-\frac{1}{12}\left(\int Q^{2}\right) a_{2}= & \int\left(\mathcal{L} V_{2}\right)\left(V_{0}-Q\right)+6 \int Q^{3}\left(1+A_{1}\right)^{2} \\
& -\frac{9}{2} a_{1} \int A_{1}^{\prime \prime} Q+\frac{3}{2} a_{1}^{2} \int Q^{\prime \prime} Q+\frac{1}{2} \int A_{1}\left(\mathcal{L} B_{1}\right)^{\prime} \\
= & 6 \int Q^{2}\left(1+A_{1}\right)^{2} V_{0}-a_{1} \int\left(\mathcal{L} B_{1}\right)^{\prime}\left(V_{0}-Q\right) \\
& -\frac{9}{2} a_{1} \int A_{1}^{\prime \prime} Q+\frac{3}{2} a_{1}^{2} \int Q^{\prime \prime} Q+\frac{1}{2} \int A_{1}\left(\mathcal{L} B_{1}\right)^{\prime} \\
= & 6 \int Q^{2}\left(1+A_{1}\right)^{2} V_{0}-a_{1} \int\left(\mathcal{L} B_{1}\right)^{\prime} V_{0} \\
& -\frac{9}{2} a_{1} \int A_{1}^{\prime \prime} Q+\frac{3}{2} a_{1}^{2} \int Q^{\prime \prime} Q+\frac{1}{2} \int A_{1}\left(\mathcal{L} B_{1}\right)^{\prime} .
\end{aligned}
$$

By inserting this value of $a_{2}$ into (8), we obtain (6). 
As $A_{1}=V_{1}-a_{1} V_{0}$ and then $\left(\mathcal{L} B_{1}\right)^{\prime}=4 Q^{3}-3 a_{1} Q^{\prime \prime}+3 A_{1}^{\prime \prime}+4 Q^{3} A_{1}$, we obtain

$$
\begin{aligned}
2 b_{2}= & 6 \int Q^{2}\left(1+A_{1}\right)^{3}-\frac{3}{2} a_{1} \int A_{1}\left(\mathcal{L} B_{1}\right)^{\prime}+\frac{9}{2} a_{1}^{2} \int A_{1} Q^{\prime \prime}-\frac{3}{2} a_{1}^{3} \int Q^{\prime \prime} Q \\
= & 6 \int Q^{2}\left(1+A_{1}\right)^{3}-\frac{3}{2} a_{1} \int A_{1}\left(4 Q^{3}-3 a_{1} Q^{\prime \prime}+3 A_{1}^{\prime \prime}+4 Q^{3} A_{1}\right) \\
& +\frac{9}{2} a_{1}^{2} \int A_{1} Q^{\prime \prime}-\frac{3}{2} a_{1}^{3} \int Q^{\prime \prime} Q \\
= & 6 \int Q^{2}\left(1+A_{1}\right)^{3}-\frac{3}{2} a_{1} \int A_{1}\left(4 Q^{3}-6 a_{1} Q^{\prime \prime}+3 A_{1}^{\prime \prime}+4 Q^{3} A_{1}\right)-\frac{3}{2} a_{1}^{3} \int Q^{\prime \prime} Q
\end{aligned}
$$

Since

$$
\mathcal{L} A_{1}+a_{1}\left(3 Q-2 Q^{4}\right)=-A_{1}^{\prime \prime}+A_{1}-4 Q^{3} A_{1}+3 a_{1} Q-2 a_{1} Q^{4}=4 Q^{3},
$$

we deduce

$$
4 Q^{3}-6 a_{1} Q^{\prime \prime}+3 A_{1}^{\prime \prime}+4 Q^{3} A_{1}=-8 Q^{3}\left(1+A_{1}\right)+3\left(A_{1}+a_{1} Q\right),
$$

and so

$$
2 b_{2}=6 \int Q^{2}\left(1+A_{1}\right)^{3}+\frac{3}{2} a_{1} \int A_{1}\left(8 Q^{3}\left(1+A_{1}\right)-3\left(A_{1}+a_{1} Q\right)\right)-\frac{3}{2} a_{1}^{3} \int Q^{\prime \prime} Q .
$$

We use again $A_{1}=V_{1}-a_{1} V_{0}$ and we sort terms by increasing powers of $a_{1}$. We get

$$
\begin{aligned}
2 b_{2}= & 6 \int Q^{2}\left(1+V_{1}-a_{1} V_{0}\right)^{3}+12 a_{1} \int\left(V_{1}-a_{1} V_{0}\right) Q^{3}\left(1+V_{1}-a_{1} V_{0}\right) \\
& -\frac{9}{2} a_{1} \int\left(V_{1}-a_{1} V_{0}\right)\left(V_{1}+a_{1}\left(-V_{0}+Q\right)\right)-\frac{3}{2} a_{1}^{3} \int Q^{\prime \prime} Q \\
= & \mu_{0}+a_{1} \mu_{1}+a_{1}^{2} \mu_{2}+a_{1}^{3} \mu_{3},
\end{aligned}
$$

where

$$
\begin{aligned}
& \mu_{0}=6 \int Q^{2}\left(1+V_{1}\right)^{3}, \\
& \mu_{1}=-18 \int Q^{2}\left(1+V_{1}\right)^{2} V_{0}+12 \int V_{1}\left(1+V_{1}\right) Q^{3}-\frac{9}{2} \int V_{1}^{2}, \\
& \mu_{2}=18 \int Q^{2}\left(1+V_{1}\right) V_{0}^{2}-12 \int V_{0}\left(1+2 V_{1}\right) Q^{3}+\frac{9}{2} \int V_{1}\left(2 V_{0}-Q\right), \\
& \mu_{3}=-6 \int Q^{2} V_{0}^{3}+12 \int Q^{3} V_{0}^{2}-\frac{9}{2} \int V_{0}^{2}+\frac{9}{2} \int V_{0} Q-\frac{3}{2} \int Q^{\prime \prime} Q .
\end{aligned}
$$

We claim the following values for $\mu_{0}, \mu_{1}, \mu_{2}$, and $\mu_{3}$. 


\section{LEMMA 1.2}

$$
\mu_{0}=-\frac{1}{9} \int Q^{2}, \quad \mu_{1}=\frac{3}{4} \int Q^{3}, \quad \mu_{2}=\frac{1}{24} \int Q, \quad \mu_{3}=\frac{5}{144} \int Q^{2} .
$$

Assuming Lemma 1.2, and using $a_{1}=-2 \int Q / \int Q^{2}$, we obtain

$$
2 b_{2}=-\frac{1}{9} \int Q^{2}-\frac{3}{2} \frac{\left(\int Q\right)\left(\int Q^{3}\right)}{\int Q^{2}}-\frac{1}{9} \frac{\left(\int Q\right)^{3}}{\left(\int Q^{2}\right)^{2}} .
$$

This completes the proof of Theorem A provided we prove Lemma 1.2.

Proof of Lemma 1.2. The main ingredient is Lemma A.1 (see appendix).

- Computation of $\mu_{0}$.

$$
\mu_{0}=6 \int Q^{2}+18 \int Q^{2} V_{1}+18 \int Q^{2} V_{1}^{2}+6 \int Q^{2} V_{1}^{3} .
$$

By Lemma A.1,

$$
\begin{aligned}
18 \int Q^{2} V_{1} & =6 \int Q^{2}\left(Q^{\prime}\left(\int_{0}^{x} Q^{2}\right)-2 Q^{3}\right) \\
& =-2 \int Q^{5}-12 \int Q^{5}=-14 \int Q^{5}=-20 \int Q^{2}, \\
18 \int Q^{2} V_{1}^{2} & =2 \int Q^{2}\left(Q^{\prime}\left(\int_{0}^{x} Q^{2}\right)-2 Q^{3}\right)^{2} \\
& =2 \int Q^{2}\left(Q^{\prime}\right)^{2}\left(\int_{0}^{x} Q^{2}\right)^{2}-8 \int Q^{5} Q^{\prime}\left(\int_{0}^{x} Q^{2}\right)+8 \int Q^{8} \\
& =2 \int Q^{4}\left(\int_{0}^{x} Q^{2}\right)^{2}-\frac{4}{5} \int Q^{7}\left(\int_{0}^{x} Q^{2}\right)^{2}+\frac{28}{3} \int Q^{8},
\end{aligned}
$$

and

$$
\begin{aligned}
6 \int Q^{2} V_{1}^{3}= & \frac{2}{9} \int Q^{2}\left(Q^{\prime}\left(\int_{0}^{x} Q^{2}\right)-2 Q^{3}\right)^{3} \\
= & \frac{2}{9} \int Q^{2}\left(Q^{\prime}\right)^{3}\left(\int_{0}^{x} Q^{2}\right)^{3}-\frac{4}{3} \int Q^{5}\left(Q^{\prime}\right)^{2}\left(\int_{0}^{x} Q^{2}\right)^{2} \\
& +\frac{8}{3} \int Q^{8} Q^{\prime}\left(\int_{0}^{x} Q^{2}\right)-\frac{16}{9} \int Q^{11} \\
= & \frac{2}{9} \int Q^{4} Q^{\prime}\left(\int_{0}^{x} Q^{2}\right)^{3}-\frac{4}{45} \int Q^{7} Q^{\prime}\left(\int_{0}^{x} Q^{2}\right)^{3}-\frac{4}{3} \int Q^{7}\left(\int_{0}^{x} Q^{2}\right)^{2} \\
& +\frac{8}{15} \int Q^{10}\left(\int_{0}^{x} Q^{2}\right)^{2}-\frac{56}{27} \int Q^{11} \\
= & -\frac{22}{15} \int Q^{7}\left(\int_{0}^{x} Q^{2}\right)^{2}+\frac{17}{30} \int Q^{10}\left(\int_{0}^{x} Q^{2}\right)^{2}-\frac{56}{11} \int Q^{11} .
\end{aligned}
$$


By Lemma A.1, $\int Q^{10}\left(\int_{0}^{x} Q^{2}\right)^{2}=\frac{35}{17} \int Q^{7}\left(\int_{0}^{x} Q^{2}\right)^{2}-\frac{10}{9 \cdot 17} \int Q^{11}$. Thus,

$$
\begin{aligned}
6 \int Q^{2} V_{1}^{3} & =-\frac{3}{10} \int Q^{7}\left(\int_{0}^{x} Q^{2}\right)^{2}-\frac{19}{9} \int Q^{11} \\
& =-\frac{3}{10} \int Q^{7}\left(\int_{0}^{x} Q^{2}\right)^{2}-\frac{40}{9} \int Q^{8} .
\end{aligned}
$$

Therefore,

$$
18 \int Q^{2} V_{1}^{2}+6 \int Q^{2} V_{1}^{3}=2 \int Q^{4}\left(\int_{0}^{x} Q^{2}\right)^{2}-\frac{11}{10} \int Q^{7}\left(\int_{0}^{x} Q^{2}\right)^{2}+\frac{44}{9} \int Q^{8} .
$$

By Lemma A.1, $\int Q^{7}\left(\int_{0}^{x} Q^{2}\right)^{2}=\frac{20}{11} \int Q^{4}\left(\int_{0}^{x} Q^{2}\right)^{2}-\frac{5}{33} \int Q^{8}$, and so

$$
18 \int Q^{2} V_{1}^{2}+6 \int Q^{2} V_{1}^{3}=\frac{91}{18} \int Q^{8} .
$$

Finally,

$$
\mu_{0}=-14 \int Q^{2}+\frac{91}{18} \int Q^{8}=-\frac{1}{9} \int Q^{2},
$$

since, by Lemma A.1, $\int Q^{8}=\frac{25}{13} \int Q^{5}=\frac{250}{7 \cdot 13} \int Q^{2}$.

- Computation of $\mu_{1}$.

$$
\mu_{1}=-18 \int Q^{2}\left(1+V_{1}\right)^{2} V_{0}+12 \int Q^{3} V_{1}\left(1+V_{1}\right)-\frac{9}{2} \int V_{1}^{2} .
$$

First,

$$
-18 \int Q^{2}\left(1+V_{1}\right)^{2} V_{0}=-18 Q^{2} V_{0}-36 \int Q^{2} V_{1} V_{0}-18 \int Q^{2} V_{1}^{2} V_{0} .
$$

We use that $V_{0}=-\frac{1}{3} Q-\frac{3}{2} x Q^{\prime}$ :

$$
\begin{aligned}
-18 Q^{2} V_{0}= & 6 \int Q^{3}+27 \int x Q^{\prime} Q^{2}=-3 \int Q^{3}, \\
-36 \int Q^{2} V_{1} V_{0}= & 4 \int Q^{2}\left(Q^{\prime}\left(\int_{0}^{x} Q^{2}\right)-2 Q^{3}\right)\left(Q+\frac{9}{2} x Q^{\prime}\right) \\
= & 4 \int Q^{3} Q^{\prime}\left(\int_{0}^{x} Q^{2}\right)+18 \int Q^{2}\left(Q^{\prime}\right)^{2} x\left(\int_{0}^{x} Q^{2}\right) \\
& -8 \int Q^{6}-36 \int x Q^{\prime} Q^{5} \\
= & -3 \int Q^{6}+18 \int x Q^{4}\left(\int_{0}^{x} Q^{2}\right)-\frac{36}{5} \int x Q^{7}\left(\int_{0}^{x} Q^{2}\right),
\end{aligned}
$$


and

$$
\begin{aligned}
-18 \int Q^{2} V_{1}^{2} V_{0}= & \frac{2}{3} \int Q^{2}\left(Q^{\prime}\left(\int_{0}^{x} Q^{2}\right)-2 Q^{3}\right)^{2}\left(Q+\frac{9}{2} x Q^{\prime}\right) \\
= & \frac{2}{3} \int\left(Q^{3}+\frac{9}{2} x Q^{\prime} Q^{2}\right) \\
& \times\left(Q^{2}\left(\int_{0}^{x} Q^{2}\right)^{2}-\frac{2}{5}\left(\int_{0}^{x} Q^{2}\right)^{2}-4 Q^{3} Q^{\prime}\left(\int_{0}^{x} Q^{2}\right)+4 Q^{6}\right) \\
= & \frac{2}{3} \int Q^{5}\left(\int_{0}^{x} Q^{2}\right)^{2}-\frac{4}{15} \int Q^{8}\left(\int_{0}^{x} Q^{2}\right)^{2}-\frac{8}{3} \int Q^{6} Q^{\prime}\left(\int_{0}^{x} Q^{2}\right) \\
& +\frac{8}{3} \int Q^{9}+3 \int x Q^{\prime} Q^{4}\left(\int_{0}^{x} Q^{2}\right)^{2}-\frac{6}{5} \int x Q^{\prime} Q^{7}\left(\int_{0}^{x} Q^{2}\right)^{2} \\
& -12 \int x Q^{7}\left(\int_{0}^{x} Q^{2}\right)+\frac{24}{5} \int x Q^{10}\left(\int_{0}^{x} Q^{2}\right)+12 \int x Q^{\prime} Q^{8} \\
= & \frac{1}{15} \int Q^{5}\left(\int_{0}^{x} Q^{2}\right)^{2}-\frac{7}{60} \int Q^{8}\left(\int_{0}^{x} Q^{2}\right)^{2}+\frac{12}{7} \int Q^{9} \\
& -\frac{66}{5} \int x Q^{7}\left(\int_{0}^{x} Q^{2}\right)+\frac{51}{10} \int x Q^{10}\left(\int_{0}^{x} Q^{2}\right) .
\end{aligned}
$$

Summing up

$$
\begin{aligned}
-18 \int Q^{2}\left(1+V_{1}\right)^{2} V_{0}= & -3 \int Q^{3}-3 \int Q^{6}+\frac{12}{7} \int Q^{9}+18 \int x Q^{4}\left(\int_{0}^{x} Q^{2}\right) \\
& -\frac{102}{5} \int x Q^{7}\left(\int_{0}^{x} Q^{2}\right)+\frac{51}{10} \int x Q^{10}\left(\int_{0}^{x} Q^{2}\right) \\
& +\frac{1}{15} \int Q^{5}\left(\int_{0}^{x} Q^{2}\right)^{2}-\frac{7}{60} \int Q^{8}\left(\int_{0}^{x} Q^{2}\right)^{2}
\end{aligned}
$$

By Lemma A.1,

$$
\int x Q^{10}\left(\int_{0}^{x} Q^{2}\right)=\frac{35}{17} \int x Q^{7}\left(\int_{0}^{x} Q^{2}\right)-\frac{80}{7 \cdot 9 \cdot 17} \int Q^{9},
$$

thus

$$
\begin{aligned}
-18 \int Q^{2}\left(1+V_{1}\right)^{2} V_{0}= & -3 \int Q^{3}-3 \int Q^{6}+\frac{4}{3} \int Q^{9}+\frac{1}{15} \int Q^{5}\left(\int_{0}^{x} Q^{2}\right)^{2} \\
& -\frac{7}{60} \int Q^{8}\left(\int_{0}^{x} Q^{2}\right)^{2}+18 \int x Q^{4}\left(\int_{0}^{x} Q^{2}\right) \\
& -\frac{99}{10} \int x Q^{7}\left(\int_{0}^{x} Q^{2}\right) .
\end{aligned}
$$

From Lemma A.1,

$$
\int x Q^{7}\left(\int_{0}^{x} Q^{2}\right)=\frac{20}{11} \int x Q^{4}\left(\int_{0}^{x} Q^{2}\right)-\frac{99 \cdot 25}{10 \cdot 11 \cdot 12} \int Q^{6},
$$


so that

$$
\begin{aligned}
-18 \int Q^{2}\left(1+V_{1}\right)^{2} V_{0}= & -3 \int Q^{3}-\frac{9}{8} \int Q^{6}+\frac{4}{3} \int Q^{9}+\frac{1}{15} \int Q^{5}\left(\int_{0}^{x} Q^{2}\right)^{2} \\
& -\frac{7}{60} \int Q^{8}\left(\int_{0}^{x} Q^{2}\right)^{2}
\end{aligned}
$$

and

$$
\begin{aligned}
12 \int V_{1}\left(1+V_{1}\right) Q^{3}= & 12 \int V_{1} Q^{3}+12 \int V_{1}^{2} Q^{3} \\
= & 4 \int\left(Q^{\prime}\left(\int_{0}^{x} Q^{2}\right)-2 Q^{3}\right) Q^{3} \\
& +\frac{4}{3} \int\left(Q^{2}\left(\int_{0}^{x} Q^{2}\right)^{2}-\frac{2}{5}\left(\int_{0}^{x} Q^{2}\right)^{2}-4 Q^{\prime} Q^{3}\left(\int_{0}^{x} Q^{2}\right)+4 Q^{6}\right) Q^{3} \\
= & -9 \int Q^{6}+\frac{128}{21} \int Q^{9}+\frac{4}{3} \int Q^{5}\left(\int_{0}^{x} Q^{2}\right)^{2} \\
& -\frac{8}{15} \int Q^{8}\left(\int_{0}^{x} Q^{2}\right)^{2}
\end{aligned}
$$

Therefore,

$$
\begin{aligned}
-18 \int Q^{2}\left(1+V_{1}\right)^{2} V_{0}+12 \int V_{1}\left(1+V_{1}\right) Q^{3}= & -3 \int Q^{3}-\frac{81}{8} \int Q^{6} \\
& +\frac{156}{21} \int Q^{9}+\frac{7}{5} \int Q^{5}\left(\int_{0}^{x} Q^{2}\right)^{2} \\
& -\frac{39}{60} \int Q^{8}\left(\int_{0}^{x} Q^{2}\right)^{2} .
\end{aligned}
$$

We use that

$$
\int Q^{8}\left(\int_{0}^{x} Q^{2}\right)^{2}=\frac{25}{13} \int Q^{5}\left(\int_{0}^{x} Q^{2}\right)^{2}-\frac{10}{7 \cdot 13} \int Q^{9}
$$

Thus,

$$
\begin{aligned}
-18 \int Q^{2}\left(1+V_{1}\right)^{2} V_{0}+12 \int V_{1}\left(1+V_{1}\right) Q^{3}= & -3 \int Q^{3}-\frac{81}{8} \int Q^{6}+\frac{15}{2} \int Q^{9} \\
& +\frac{3}{20} \int Q^{5}\left(\int_{0}^{x} Q^{2}\right)^{2} \\
= & -3 \int Q^{3}+\frac{39}{8} \int Q^{6} \\
& +\frac{3}{20} \int Q^{5}\left(\int_{0}^{x} Q^{2}\right)^{2}
\end{aligned}
$$

since $\int Q^{9}=2 \int Q^{6}$ (Lemma A.1). 


$$
\begin{aligned}
-\frac{9}{2} \int V_{1}^{2} & =-\frac{1}{2} \int\left(Q^{\prime}\left(\int_{0}^{x} Q^{2}\right)^{2}-2 Q^{3}\right)^{2} \\
& =-\frac{1}{2} \int Q^{2}\left(\int_{0}^{x} Q^{2}\right)^{2}+\frac{1}{5} \int Q^{5}\left(\int_{0}^{x} Q^{2}\right)^{2}+2 \int Q^{\prime} Q^{3}\left(\int_{0}^{x} Q^{2}\right)-2 \int Q^{6} \\
& =-\frac{1}{2} \int Q^{2}\left(\int_{0}^{x} Q^{2}\right)^{2}+\frac{1}{5} \int Q^{5}\left(\int_{0}^{x} Q^{2}\right)^{2}-\frac{5}{2} \int Q^{6} .
\end{aligned}
$$

Summing up, we get

$$
\mu_{1}=-3 \int Q^{3}+\frac{19}{6} \int Q^{6}-\frac{1}{2} \int Q^{2}\left(\int_{0}^{x} Q^{2}\right)^{2}+\frac{7}{20} \int Q^{5}\left(\int_{0}^{x} Q^{2}\right)^{2},
$$

but

$$
\int Q^{5}\left(\int_{0}^{x} Q^{2}\right)^{2}=\frac{10}{7} \int Q^{2}\left(\int_{0}^{x} Q^{2}\right)^{2}-\frac{5}{14} \int Q^{6},
$$

and $\int Q^{6}=\frac{5}{3} \int Q^{3}$, by Lemma A.1, so that

$$
\mu_{1}=\frac{3}{4} \int Q^{3}
$$

- Computation of $\mu_{2}$.

$$
\mu_{2}=18 \int Q^{2}\left(1+V_{1}\right) V_{0}^{2}-12 \int Q^{3} V_{0}\left(1+2 V_{1}\right)+\frac{9}{2} \int V_{1}\left(2 V_{0}-Q\right) .
$$

First,

$$
\begin{aligned}
18 \int Q^{2}\left(1+V_{1}\right) V_{0}^{2}= & \frac{2}{3} \int Q^{2}\left(3+Q^{\prime}\left(\int_{0}^{x} Q^{2}\right)-2 Q^{3}\right)\left(Q+\frac{9}{2} x Q^{\prime}\right)^{2} \\
= & \frac{2}{3} \int\left(Q^{4}+9 x Q^{3} Q^{\prime}+\frac{81}{4} x^{2} Q^{4}-\frac{81}{10} x^{2} Q^{7}\right) \\
& \times\left(3+Q^{\prime}\left(\int_{0}^{x} Q^{2}\right)-2 Q^{3}\right) \\
= & 2 \int Q^{4}+18 \int x Q^{3} Q^{\prime}+\frac{81}{2} \int x^{2} Q^{4} \\
& -\frac{81}{4} \int x^{2} Q^{7}+\frac{2}{3} \int Q^{4} Q^{\prime}\left(\int_{0}^{x} Q^{2}\right) \\
& +6 \int x Q^{5}\left(\int_{0}^{x} Q^{2}\right)-\frac{12}{5} \int x Q^{8}\left(\int_{0}^{x} Q^{2}\right) \\
& +\frac{27}{2} \int x^{2} Q^{4} Q^{\prime}\left(\int_{0}^{x} Q^{2}\right)-\frac{27}{5} \int x^{2} Q^{7} Q^{\prime}\left(\int_{0}^{x} Q^{2}\right)-\frac{4}{3} \int Q^{7} \\
& -12 \int x Q^{6} Q^{\prime}-27 \int x^{2} Q^{7}+\frac{54}{5} \int x^{2} Q^{10} .
\end{aligned}
$$


Thus,

$$
\begin{aligned}
18 \int Q^{2}\left(1+V_{1}\right) V_{0}^{2}= & -\frac{5}{2} \int Q^{4}+\left(-\frac{22}{15}+\frac{12}{7}\right) \int Q^{7}+\frac{81}{2} \int x^{2} Q^{4}-\frac{459}{10} \int x^{2} Q^{7} \\
& +\frac{17 \cdot 27}{40} \int x^{2} Q^{10}+\frac{3}{5} \int x Q^{5}\left(\int_{0}^{x} Q^{2}\right)-\frac{21}{20} \int x Q^{8}\left(\int_{0}^{x} Q^{2}\right) .
\end{aligned}
$$

But (Lemma A.1)

and

$$
\int x^{2} Q^{10}=\frac{35}{17} \int x^{2} Q^{7}-\frac{10}{7 \cdot 17} \int Q^{7}
$$

$$
\int x^{2} Q^{7}=\frac{20}{11} \int x^{2} Q^{4}-\frac{5}{22} \int Q^{4}
$$

so that

$$
-\frac{459}{10} \int x^{2} Q^{7}+\frac{17 \cdot 27}{40} \int x^{2} Q^{10}=-\frac{81}{2} \int x^{2} Q^{4}+\frac{81}{16} \int Q^{4}-\frac{27}{28} \int Q^{7} .
$$

Therefore,

$$
\begin{aligned}
18 \int Q^{2}\left(1+V_{1}\right) V_{0}^{2}= & \frac{41}{16} \int Q^{4}-\frac{43}{60} \int Q^{7} \\
& +\frac{3}{5} \int x Q^{5}\left(\int_{0}^{x} Q^{2}\right)-\frac{21}{20} \int x Q^{8}\left(\int_{0}^{x} Q^{2}\right), \\
-12 \int V_{0}\left(1+2 V_{1}\right) Q^{3}= & 4 \int\left(Q+\frac{9}{2} x Q^{\prime}\right) Q^{3}\left(1+\frac{2}{3} Q^{\prime}\left(\int_{0}^{x} Q^{2}\right)-\frac{4}{3} Q^{3}\right) \\
= & 4 \int\left(Q^{4}+\frac{9}{2} x Q^{\prime} Q^{3}\right)+\frac{8}{3} \int Q^{4} Q^{\prime}\left(\int_{0}^{x} Q^{2}\right) \\
& +12 \int x Q^{5}\left(\int_{0}^{x} Q^{2}\right)-\frac{24}{5} \int x Q^{8}\left(\int_{0}^{x} Q^{2}\right)-\frac{16}{3} \int Q^{7} \\
& -24 \int x Q^{6} Q^{\prime} \\
= & -\frac{1}{2} \int Q^{4}-\frac{8}{15} \int Q^{7}+12 \int x Q^{5}\left(\int_{0}^{x} Q^{2}\right) \\
& -\frac{24}{5} \int x Q^{8}\left(\int_{0}^{x} Q^{2}\right)-\frac{40}{21} \int Q^{7} .
\end{aligned}
$$

Thus,

$$
\begin{aligned}
18 \int Q^{2}(1+ & \left.V_{1}\right) V_{0}^{2}-12 \int V_{0}\left(1+2 V_{1}\right) Q^{3} \\
& =\frac{33}{16} \int Q^{4}-\frac{265}{84} \int Q^{7}+\frac{63}{5} \int x Q^{5}\left(\int_{0}^{x} Q^{2}\right)-\frac{117}{20} \int x Q^{8}\left(\int_{0}^{x} Q^{2}\right) .
\end{aligned}
$$


But, by Lemma A.1,

$$
\int x Q^{8}\left(\int_{0}^{x} Q^{2}\right)=\frac{25}{13} \int x Q^{5}\left(\int_{0}^{x} Q^{2}\right)-\frac{12}{91} \int Q^{7},
$$

and so

$$
\begin{aligned}
18 \int Q^{2}\left(1+V_{1}\right) V_{0}^{2}-12 \int V_{0}(1+ & \left.2 V_{1}\right) Q^{3} \\
& =\frac{33}{16} \int Q^{4}-\frac{143}{60} \int Q^{7}+\frac{27}{20} \int x Q^{5}\left(\int_{0}^{x} Q^{2}\right) .
\end{aligned}
$$

As $\int Q^{7}=\frac{20}{11} \int Q^{4}$, we get

$$
18 \int Q^{2}\left(1+V_{1}\right) V_{0}^{2}-12 \int V_{0}\left(1+2 V_{1}\right) Q^{3}=-\frac{109}{48} \int Q^{4}+\frac{27}{20} \int x Q^{5}\left(\int_{0}^{x} Q^{2}\right)
$$

and

$$
\begin{aligned}
\frac{9}{2} \int V_{1}\left(2 V_{0}-Q\right)= & -\frac{1}{2} \int\left(Q^{\prime}\left(\int_{0}^{x} Q^{2}\right)-2 Q^{3}\right)\left(5 Q+9 x Q^{\prime}\right) \\
= & -\frac{5}{2} \int Q Q^{\prime}\left(\int_{0}^{x} Q^{2}\right)-\frac{9}{2} \int x Q^{2}\left(\int_{0}^{x} Q^{2}\right)+\frac{9}{5} \int x Q^{5}\left(\int_{0}^{x} Q^{2}\right) \\
& +5 \int Q^{4}+9 \int x Q^{3} Q^{\prime} \\
= & 4 \int Q^{4}-\frac{9}{2} \int x Q^{2}\left(\int_{0}^{x} Q^{2}\right)+\frac{9}{5} \int x Q^{5}\left(\int_{0}^{x} Q^{2}\right) .
\end{aligned}
$$

Finally,

$$
\mu_{2}=\frac{83}{48} \int Q^{4}+\frac{63}{20} \int x Q^{5}\left(\int_{0}^{x} Q^{2}\right)-\frac{9}{2} \int x Q^{2}\left(\int_{0}^{x} Q^{2}\right)
$$

But, by Lemma A.1,

$$
\frac{63}{20} \int x Q^{5}\left(\int_{0}^{x} Q^{2}\right)=\frac{9}{2} \int x Q^{2}\left(\int_{0}^{x} Q^{2}\right)-\frac{27}{16} \int Q^{4}
$$

Thus

$$
\mu_{2}=\frac{1}{24} \int Q^{4}=\frac{1}{24} \int Q
$$

- Computation of $\mu_{3}$.

$$
\mu_{3}=-6 \int Q^{2} V_{0}^{3}+12 \int Q^{3} V_{0}^{2}-\frac{9}{2} \int V_{0}^{2}+\frac{9}{2} \int V_{0} Q-\frac{3}{2} \int Q^{\prime \prime} Q .
$$


First,

$$
\begin{aligned}
-6 \int Q^{2} V_{0}^{3}+12 \int Q^{3} V_{0}^{2}= & 6 \int V_{0}^{2}\left(-V_{0}+2 Q\right) \\
= & \frac{2}{9} \int\left(Q+\frac{9}{2} x Q^{\prime}\right)^{2} Q^{2}\left(7 Q+\frac{9}{2} x Q^{\prime}\right) \\
= & \frac{2}{9} \int\left(Q^{2}+9 x Q^{\prime} Q+\frac{81}{4} x^{2} Q^{2}-\frac{81}{10} x^{2} Q^{5}\right) \\
& \times\left(7 Q^{3}+\frac{9}{2} x Q^{2} Q^{\prime}\right) \\
= & \frac{14}{9} \int Q^{5}+\int x Q^{4} Q^{\prime}+14 \int x Q^{4} Q^{\prime}+9 \int x^{2} Q^{5} \\
& -\frac{18}{5} \int x^{2} Q^{8}+\frac{63}{2} \int x^{2} Q^{5}+\frac{81}{4} \int x^{3} Q^{4} Q^{\prime} \\
& -\frac{63}{5} \int x^{2} Q^{8}-\frac{81}{10} \int x^{3} Q^{7} Q^{\prime} \\
= & -\frac{13}{9} \int Q^{5}+\frac{567}{20} \int x^{2} Q^{5}-\frac{1053}{80} \int x^{2} Q^{8} .
\end{aligned}
$$

But, by Lemma A.1,

$$
\int x^{2} Q^{8}=\frac{25}{13} \int x^{2} Q^{5}-\frac{2}{13} \int Q^{5}
$$

and so

$$
-6 \int Q^{2} V_{0}^{3}+12 \int Q^{3} V_{0}^{2}=\frac{209}{360} \int Q^{5}+\frac{243}{80} \int x^{2} Q^{5} .
$$

Second,

$$
\begin{aligned}
\frac{9}{2} \int V_{0}\left(-V_{0}+Q\right) & =-\frac{1}{2} \int\left(Q+\frac{9}{2} x Q^{\prime}\right)\left(4 Q+\frac{9}{2} x Q^{\prime}\right) \\
& =-\frac{1}{2} \int\left(4 Q^{2}+\frac{9}{2} x Q Q^{\prime}+18 x Q^{\prime} Q+\frac{81}{4} x^{2} Q^{2}-\frac{81}{10} x^{2} Q^{5}\right) \\
& =\frac{29}{8} \int Q^{2}-\frac{81}{8} \int x^{2} Q^{2}+\frac{81}{20} \int x^{2} Q^{5} .
\end{aligned}
$$

Thus,

$$
\mu_{3}=\frac{29}{8} \int Q^{2}+\frac{209}{360} \int Q^{5}-\frac{81}{8} \int x^{2} Q^{2}+\frac{567}{80} \int x^{2} Q^{5} .
$$

But

$$
\int x^{2} Q^{5}=\frac{10}{7} \int x^{2} Q^{2}-\frac{5}{7} \int Q^{2} \quad \text { and } \quad \int Q^{5}=\frac{10}{7} \int Q^{2} \text {, }
$$

so that

$$
\mu_{3}=\frac{5}{144} \int Q^{2} .
$$




\section{Resolution of systems $\left(\Omega_{2,0}\right)$ and $\left(\Omega_{1,1}\right)$ for $p=2$}

In this section, we consider the equation

$$
\partial_{t} u+\partial_{x}\left(\partial_{x}^{2} u+u^{2}\right)=0, \quad x, t \in \mathbb{R} .
$$

The problem is similar, except that there exist explicit multi-solitons which describe the collision of several solitons. Our objective in [10] was to point out that at the main orders our decomposition corresponds to the explicit 2-soliton solution. In doing so, we needed to compute the solutions of $\left(\Omega_{1,0}\right),\left(\Omega_{1,1}\right)$ and $\left(\Omega_{2,0}\right)$ (for the nonlinearity $u^{2}$, the system $\left(\Omega_{1,1}\right)$ appears at the same order of size as $\left.\left(\Omega_{2,0}\right)\right)$. Note that the same could be done for $u^{3}$.

In the case $u^{2}$,

$$
Q(x)=\frac{3}{2} \cosh ^{-1}\left(\frac{x}{2}\right) \quad \text { solves } \quad Q^{\prime \prime}+Q=Q^{2} .
$$

We recall from [10, Lemma 3.1] that the resolution of $\left(\Omega_{1,0}\right)$ gives

$$
a_{1,0}=\frac{2}{3}, \quad A_{1,0}=-\frac{4}{3} Q, \quad B_{1,0}=2 \frac{Q^{\prime}}{Q} .
$$

We now solve $\left(\Omega_{2,0}\right)$ and $\left(\Omega_{1,1}\right)$.

Proposition 2.1 (Resolution of $\left(\Omega_{2,0}\right)$ and $\left(\Omega_{1,1}\right)$ for $p=2$ ).

$a_{2,0}=-\frac{4}{9}, \quad a_{1,1}=\frac{2}{3}, A_{2,0}=-2+\frac{4}{3} Q, \quad A_{1,1}=2-\frac{2}{3} Q-\frac{1}{3} x Q^{\prime}, \quad b_{2,0}=\lim _{+\infty} B_{2,0}=\frac{4}{3}$.

for solutions to systems $\left(\Omega_{2,0}\right)$ and $\left(\Omega_{1,1}\right)$.

From [10, Corollary 3.1], there are several solutions. Indeed, in general, if $\left(a_{k, \ell}, A_{k, \ell}, B_{k, \ell}\right)$ is solution of $\left(\Omega_{k, \ell}\right)$, then for any $\left(\gamma_{k, \ell}, \delta_{k, \ell}\right) \in \mathbb{R}^{2}$,

$$
\left(a_{k, \ell}+\gamma_{k, \ell} a_{1,0}, A_{k, \ell}+\gamma_{k, \ell}\left(1+A_{1,0}\right), B_{k, \ell}+\gamma_{k, \ell} B_{1,0}+\delta_{k, \ell} Q^{\prime}\right)
$$

is also solution, which gives two degrees of freedom at each step. The choice of the solution above is related to the exact 2 -soliton solutions.

Proof of Proposition 2.1.

- System $\left(\Omega_{2,0}\right)$.

From [10, Proposition 2.1]

$$
\left\{\begin{aligned}
\left(\mathcal{L} A_{2,0}\right)^{\prime}+a_{2,0}\left(3 Q-2 Q^{2}\right)^{\prime}= & \left(-A_{1,0}+A_{1,0}^{2}\right)^{\prime}-\left(3 B_{1,0}^{\prime \prime}+2 Q B_{1,0}\right) \\
& -a_{1,0}\left(Q+3 A_{1,0}^{\prime \prime}+2 Q A_{1,0}\right)^{\prime}+3 a_{1,0}^{2} Q^{(3)} \\
\left(\mathcal{L} B_{2,0}\right)^{\prime}+3 a_{2,0} Q^{\prime \prime}-3 A_{2,0}^{\prime \prime}- & 2 Q A_{2,0} \\
= & A_{1,0}+A_{1,0}^{2}+\left(-2 B_{1,0}+A_{1,0} B_{1,0}\right)^{\prime} \\
& -\frac{1}{2} a_{1,0}\left(9 A_{1,0}^{\prime}+3 B_{1,0}^{\prime \prime}+2 Q B_{1,0}\right)^{\prime}+\frac{3}{2} a_{1,0}^{2} Q^{\prime \prime}
\end{aligned}\right.
$$


Replacing $A_{1,0}$ and $B_{1,0}$, the first line of the system becomes

$$
\begin{aligned}
\left(\mathcal{L} A_{2,0}\right)^{\prime}= & \left(\frac{4}{3} Q+\frac{16}{9} Q^{2}\right)^{\prime}-\left(-2 Q^{\prime}+4 Q^{\prime} Q Q^{\prime}\right) \\
& -\frac{2}{3}\left(Q-4 Q^{\prime \prime}-\frac{8}{3} Q^{2}\right)^{\prime}+\frac{4}{3} Q^{(3)}-a_{2,0}\left(3 Q-2 Q^{2}\right)^{\prime} \\
= & \left(\frac{8}{3} Q-\frac{4}{9} Q^{2}-a_{2,0}\left(3 Q-2 Q^{2}\right)\right)^{\prime} .
\end{aligned}
$$

We choose $\gamma_{2,0}=-2$ in (10), so that we consider $A_{2,0}$ solution of

$$
\mathcal{L} A_{2,0}=\frac{8}{3} Q-\frac{4}{9} Q^{2}-a_{2,0}\left(3 Q-2 Q^{2}\right)-2 .
$$

Consider the function $V_{2}$ defined by

$$
V_{2}=-2+\frac{16}{9} Q+\frac{2}{3} x Q^{\prime}, \quad \mathcal{L} V_{2}=\frac{8}{3} Q-\frac{4}{9} Q^{2}-2 .
$$

Then, $A_{2,0}=V_{2}-a_{2,0} V_{0}$, where in this case $V_{0}=-Q-\frac{3}{2} x Q^{\prime}$ solves $\mathcal{L} V_{0}=3 Q-2 Q^{2}$. Inserting the expressions of $a_{1,0}, A_{1,0}, B_{1,0}$, and $A_{2,0}$ into the second line of $\left(\Omega_{2,0}\right)$ and after some calculations, we obtain the following equation for $B_{2,0}$ :

$$
\left(\mathcal{L} B_{2,0}\right)^{\prime}+a_{2,0} Z_{0}=3 V_{2}^{\prime \prime}+2 Q V_{2}+\frac{4}{3} Q+\frac{4}{9} Q^{2},
$$

where $Z_{0}=3 Q^{\prime \prime}+3 V_{0}^{\prime \prime}+2 Q V_{0}$. The value of $a_{2,0}$ is thus given by

$$
a_{2,0} \int Z_{0} Q=\int Z_{2} Q
$$

where $Z_{2}=3 V_{2}^{\prime \prime}+2 Q V_{2}+\frac{4}{3} Q+\frac{4}{9} Q^{2}$. Recall that $\int Z_{0} Q=-\frac{3}{4} \int Q^{2}$ [10, Claim 2.3].

We claim

$$
\int Z_{2} Q=\frac{1}{3} \int Q
$$

Proof of (11).

$$
\begin{aligned}
\int Z_{2} Q & =\int V_{2}\left(3 Q^{\prime \prime}+2 Q^{2}\right)+\frac{4}{3} \int Q^{2}+\frac{4}{9} \int Q^{3} \\
& =\int \mathcal{L} V_{2}\left(-2 Q-\frac{3}{2} x Q^{\prime}\right)+\frac{4}{3} \int Q^{2}+\frac{4}{9} \int Q^{3} \\
& =-\int\left(\frac{8}{3} Q-\frac{4}{9} Q^{2}-2\right)\left(2 Q+\frac{3}{2} x Q^{\prime}\right)+\frac{4}{3} \int Q^{2}+\frac{4}{9} \int Q^{3} \\
& =\int Q-2 \int Q^{2}+\frac{10}{9} \int Q^{3}=\frac{1}{3} \int Q
\end{aligned}
$$

Thus,

$$
a_{2,0}=-\frac{4}{9} \quad \text { and } \quad A_{2,0}=-2+\frac{4}{3} Q .
$$


Finally, we compute $b_{2,0}$. As in section 1 , we have $2 b_{2,0}=\int\left(\mathcal{L} B_{2,0}\right)^{\prime}$. Thus, since $\int Q=6, \int Q V_{0}=-\frac{1}{4} \int Q$, and $\int V_{2} Q=-\frac{5}{9} \int Q$, we obtain

$$
2 b_{2,0}=-2 a_{2,0} \int V_{0} Q+2 \int V_{2} Q+\frac{4}{3} \int Q+\frac{4}{9} \int Q^{2}=\frac{4}{9} \int Q=\frac{8}{3} .
$$

- $\operatorname{System}\left(\Omega_{1,1}\right)$.

The system $\left(\Omega_{1,1}\right)$ writes

$$
\left\{\begin{array}{l}
\left(\mathcal{L} A_{1,1}\right)^{\prime}+a_{1,1}\left(3 Q-2 Q^{2}\right)^{\prime}=3 A_{1,0}^{\prime}+3 B_{1,0}^{\prime \prime}+2 Q B_{1,0} \\
\left(\mathcal{L} B_{1,1}\right)^{\prime}+3 a_{1,1} Q^{\prime \prime}-3 A_{1,1}^{\prime \prime}-2 Q A_{1,1}=3 B_{1,0}^{\prime}
\end{array}\right.
$$

Replacing $A_{1,0}$ and $B_{1,0}$ by their respective expressions, the first line of the system becomes

$$
\left(\mathcal{L} A_{1,1}\right)^{\prime}=-2 Q^{\prime}-a_{1,1}\left(3 Q-2 Q^{2}\right)^{\prime} .
$$

We choose $\gamma_{1,1}=2$, so that

$$
\mathcal{L} A_{1,1}=2-2 Q-a_{1,1}\left(3 Q-2 Q^{2}\right) \quad \text { and } \quad A_{1,1}=2-2 Q-x Q^{\prime}-a_{1,1} V_{0} .
$$

Thus, the second line of the system becomes

$$
\left(\mathcal{L} B_{1,1}\right)^{\prime}+a_{1,1} Z_{0}=-2 Q+3\left(-2 Q-x Q^{\prime}\right)^{\prime \prime}+2 Q\left(2-2 Q-x Q^{\prime}\right),
$$

and so

$$
a_{1,1} \int Z_{0} Q=-\frac{5}{2} \int Q^{2}+\frac{5}{3} \int Q^{3}=-\frac{1}{2} \int Q^{2}=-\frac{1}{2} \int Q .
$$

Since $\int Z_{0} Q=-\frac{3}{4} \int Q$, we deduce $a_{1,1}=\frac{2}{3}$ and so $A_{1,1}=2-\frac{2}{3} Q-\frac{1}{3} x Q^{\prime}$.

\section{Appendix: Identities for solutions of (2)}

Lemma A.1. Let $Q$ be defined in (2). For any $r \geq 1$,

$$
\begin{aligned}
\int Q^{r+3} & =\frac{5 r}{2 r+3} \int Q^{r} \\
\int Q^{r+3}\left(\int_{0}^{x} Q^{2}\right)^{2} & =\frac{5 r}{2 r+3} \int Q^{r}\left(\int_{0}^{x} Q^{2}\right)^{2}-\frac{10}{(2 r+3)(r+2)} \int Q^{r+4}, \\
\int x Q^{r+3} \int_{0}^{x} Q^{2} & =\frac{5 r}{2 r+3} \int x Q^{r} \int_{0}^{x} Q^{2}-\frac{10(r+1)}{(2 r+3) r(r+2)} \int Q^{r+2} \\
\int x^{2} Q^{r+3} & =\frac{5 r}{2 r+3} \int x^{2} Q^{r}-\frac{10}{r(2 r+3)} \int Q^{r} .
\end{aligned}
$$


Proof. To prove this lemma, we only use

$$
Q^{4}=Q-Q^{\prime \prime} \quad \text { and } \quad\left(Q^{\prime}\right)^{2}=Q^{2}-\frac{2}{5} Q^{5} .
$$

Proof of (12). By integration by parts,

$$
\begin{aligned}
\int Q^{r+3} & =\int Q^{r-1} Q^{4}=\int Q^{r-1}\left(Q-Q^{\prime \prime}\right)=\int Q^{r}+(r-1) \int\left(Q^{\prime}\right)^{2} Q^{r-2} \\
& =r \int Q^{r}-\frac{2(r-1)}{5} \int Q^{r+3} .
\end{aligned}
$$

Thus,

$$
\frac{2 r+3}{5} \int Q^{r+3}=r \int Q^{r} .
$$

Proof of (13). By similar arguments,

$$
\begin{aligned}
\int Q^{r+3}\left(\int_{0}^{x} Q^{2}\right)^{2}= & \int Q^{r-1} Q^{4}\left(\int_{0}^{x} Q^{2}\right)^{2}=\int Q^{r-1}\left(Q-Q^{\prime \prime}\right)\left(\int_{0}^{x} Q^{2}\right)^{2} \\
= & \int Q^{r}\left(\int_{0}^{x} Q^{2}\right)^{2}+(r-1) \int\left(Q^{\prime}\right)^{2} Q^{r-2}\left(\int_{0}^{x} Q^{2}\right)^{2} \\
& +2 \int Q^{r+1} Q^{\prime} \int_{0}^{x} Q^{2} \\
= & r \int Q^{r}\left(\int_{0}^{x} Q^{2}\right)^{2}-\frac{2(r-1)}{5} \int Q^{r+3}\left(\int_{0}^{x} Q^{2}\right)^{2}-\frac{2}{r+2} \int Q^{r+4}
\end{aligned}
$$

Thus,

$$
\frac{2 r+3}{5} \int Q^{r+3}\left(\int_{0}^{x} Q^{2}\right)^{2}=r \int Q^{r}\left(\int_{0}^{x} Q^{2}\right)^{2}-\frac{2}{r+2} \int Q^{r+4} .
$$

Proof of (14).

$$
\begin{aligned}
\int x Q^{r+3} \int_{0}^{x} Q^{2}= & \int x Q^{r-1} Q^{4} \int_{0}^{x} Q^{2}=\int x Q^{r-1}\left(Q-Q^{\prime \prime}\right) \int_{0}^{x} Q^{2} \\
= & \int x Q^{r} \int_{0}^{x} Q^{2}+(r-1) \int x\left(Q^{\prime}\right)^{2} Q^{r-2} \int_{0}^{x} Q^{2}+\int Q^{r-1} Q^{\prime} \int_{0}^{x} Q^{2} \\
& +\int x Q^{r+1} Q^{\prime} \\
= & r \int x Q^{r} \int_{0}^{x} Q^{2}-\frac{2(r-1)}{5} \int x Q^{r+3} \int_{0}^{x} Q^{2}-\left(\frac{1}{r}+\frac{1}{r+2}\right) \int Q^{r+2} .
\end{aligned}
$$

Thus,

$$
\frac{2 r+3}{5} \int x Q^{r+3} \int_{0}^{x} Q^{2}=r \int x Q^{r} \int_{0}^{x} Q^{2}-\frac{2(r+1)}{r(r+2)} \int Q^{r+2} .
$$


Proof of (15).

$$
\begin{aligned}
\int x^{2} Q^{r+3}= & \int x^{2} Q^{r-1}\left(Q-Q^{\prime \prime}\right)=\int x^{2} Q^{r}+(r-1) \int x^{2}\left(Q^{\prime}\right)^{2} Q^{r-2} \\
& +2 \int x Q^{r-1} Q^{\prime} \\
= & r \int x^{2} Q^{r}-\frac{2(r-1)}{5} \int x^{2} Q^{r+3}-\frac{2}{r} \int Q^{r} .
\end{aligned}
$$

Thus,

$$
\frac{2 r+3}{5} \int x^{2} Q^{r+3}=r \int x^{2} Q^{r}-\frac{2}{r} \int Q^{r} .
$$

Proof of (9). This is [10, Claim 2.3]. We repeat it here for convenience.

We compute $\int Z_{0} Q$, where $Z_{0}=3 Q^{\prime \prime}+3 V_{0}^{\prime \prime}+4 Q^{3} V_{0}$. By $Q^{\prime \prime}=Q-Q^{4}$, we get:

$$
\begin{aligned}
\int Z_{0} Q & =\int\left(3 Q^{\prime \prime}+3 V_{0}^{\prime \prime}+4 Q^{3} V_{0}\right) Q=3 \int Q^{2}-3 \int Q^{5}+\int V_{0}\left(3 Q^{\prime \prime}+4 Q^{4}\right) \\
& =3 \int Q^{2}-3 \int Q^{5}+\int V_{0}\left(3 Q+Q^{4}\right) .
\end{aligned}
$$

We compute the last term, integrating by parts:

$$
\int V_{0}\left(3 Q+Q^{4}\right)=-\int\left(\frac{1}{3} Q+\frac{3}{2} x Q^{\prime}\right)\left(3 Q+Q^{4}\right)=\frac{5}{4} \int Q^{2}-\frac{1}{30} \int Q^{5} .
$$

Finally, using Lemma A.1,

$$
\int Z_{0} Q=\frac{17}{4} \int Q^{2}-\frac{91}{30} \int Q^{5}=-\frac{1}{12} \int Q^{2}
$$

\section{References}

[1] J. L. Bona, W. G. Pritchard, and L. R. Scott, Solitary-wave interaction, Phys. Fluids 23 (1980), 438-441.

[2] W. Craig, P. Guyenne, J. Hammack, D. Henderson, and C. Sulem, Solitary water wave interactions, Phys. Fluids 18 (2006), no. 5, 057106, 25.

[3] E. Fermi, J. Pasta, and S. Ulam, Studies of nonlinear problems, I, Los Alamos Report LA1940 (1955); reprinted in A. C. Newell (ed.), Nonlinear wave motion, Lectures in Applied Mathematics, vol. 15, American Mathematical Society, Providence, R.I., 1974.

[4] R. Hirota, Exact solution of the Korteweg-de Vries equation for multiple collisions of solitons, Phys. Rev. Lett. 27 (1971), 1192-1194.

[5] P. D. Lax, Integrals of nonlinear equations of evolution and solitary waves, Comm. Pure Appl. Math. 21 (1968), 467-490.

[6] Y. Li and D. H. Sattinger, Soliton collisions in the ion acoustic plasma equations, J. Math. Fluid Mech. 1 (1999), no. 1, 117-130. 
[7] Y. Martel, Asymptotic $N$-soliton-like solutions of the subcritical and critical generalized Korteweg-de Vries equations, Amer. J. Math. 127 (2005), no. 5, 1103-1140.

[8] Y. Martel, F. Merle, and T.-P. Tsai, Stability and asymptotic stability in the energy space of the sum of $N$ solitons for subcritical gKdV equations, Comm. Math. Phys. 231 (2002), no. 2, $347-373$.

[9] Y. Martel and F. Merle, Refined asymptotics around solitons for gKdV equations, Discrete Contin. Dyn. Syst. 20 (2008), no. 2, 177-218.

[10] Description of two soliton collision for the quartic KdV equation, preprint, available at arXiv:0709.2672v1.

[11] R. M. Miura, The Korteweg-de Vries equation: A survey of results, SIAM Rev. 18 (1976), no. 3, $412-459$.

[12] M. Wadati and M. Toda, The exact $N$-soliton solution of the Korteweg-de Vries equation, J. Phys. Soc. Japan 32 (1972), 1403-1411.

[13] N. J. Zabusky, Solitons and energy transport in nonlinear lattices, Comput. Phys. Comm. 5 (1973), 1-10.

[14] N. J. Zabusky and M. D. Kruskal, Interaction of "solitons" in a collisionless plasma and recurrence of initial states, Phys. Rev. Lett. 15 (1965), 240-243. 